\title{
Óbitos por Anomalias Congênitas do Coração e Circulatório no Estado do Pará nos anos de 2007 a 2017: Revisão Sistemática da Literatura
}

\author{
Eliane Moura da Silva ${ }^{1}$, Antônia Gomes de Olinda ${ }^{2}$, Francisco Hilângelo Vieira Barros ${ }^{3}$, \\ Maria Alcione Silva Gomes Roseno ${ }^{4}$
}

\begin{abstract}
Resumo: As cardiopatias representam importantes causas de internação e óbitos em crianças e o conhecimento de informações de âmbito epidemiológico são determinantes para tomada de decisão nos serviços de saúde. O objetivo do presente estudo é analisar o número de óbitos por causas evitáveis, desde o ano de 2007 até 2017, sobre anomalias congênitas do coração e circulatório em crianças menores de cinco anos no estado do Pará. A pesquisa é do tipo qualitativa, descritivo-exploratória. Foi realizada uma análise documental, contidos nas declarações de óbitos, levantados pelo Sistema de Informação sobre Mortalidade (SIM) e a busca na literatura para identificar a importância da assistência de enfermagem no cuidado de crianças menores de cinco com anomalias congênitas do coração e circulatório. O estado do Pará ocupa, no ranking, o primeiro lugar (44,94\%) em relação à região norte, embora o índice seja considerado baixo se comparado aos outros estados e regiões. Nas crianças menores de cinco anos, as mortes devido a malformações estão entre as principais causas de óbito, atrás apenas das afecções perinatais. A importância da assistência de enfermagem no cuidado dessas crianças possui uma tarefa desgastante do ponto de vista emocional, mas capaz de gerar grande satisfação pessoal. Acredita-se ser de grande importância a realização de pesquisas de enfermagem com famílias que vivenciam tal situação, a fim de melhor compreender sua experiência no processo de cuidar da criança em seu cotidiano.
\end{abstract}

Palavras-Chave: Anomalias congênitas. Enfermagem Pediátrica. Saúde da criança.

\section{Objectives for Congenital Anomalies of Heart and Circulatory in the State of Pará in the Years of 2007 to 2017: Systematic Review of Literature}

\begin{abstract}
Heart diseases represent important causes of hospitalization and deaths in children and the knowledge of information of epidemiological scope are determinant for decision making in the health services. The objective of the present study is to analyze the number of deaths due to preventable causes, from 2007 to 2017, on congenital heart and circulatory abnormalities in children under five in the state of Pará. The research is qualitative, descriptive-exploratory. A documentary analysis was carried out, contained in the death certificates, collected by the Mortality Information System (SIM) and the literature search to identify the importance of nursing care in the care of children under five with congenital heart and circulatory abnormalities. The state of Pará occupies, in the ranking, the first place (44.94\%) in relation to the north region, although the index is considered low when compared to the other states and regions. In children under five, deaths due to malformations are among the leading causes of death, behind only perinatal conditions. The importance of nursing care in the care of these children has an emotionally demanding task, but capable of generating great personal satisfaction. It is believed to be of great importance to conduct nursing research with families who experience such a situation in order to better understand their experience in the process of caring for the child in their daily lives.
\end{abstract}

Keywords: Congenital anomalies. Pediatric Nursing. Child health.

\footnotetext{
${ }^{1}$ Enfermeira. Mestre em Epidemiologia e Vigilância em Saúde pelo Instituto Evandro Chagas, Brasil. E-mail:eliane82moura@ hotmail.com;

${ }^{2}$ Especialista em Enfermagem em UTI Pediátrica e Neonatal pela Faculdade Unyleya, Brasília-DF. Enfermeira do Hospital

Universitário da Grande Dourados-MS;

${ }^{3}$ Mestre em Saúde da Criança e do Adolescente pela Universidade Estadual do Ceará, Fortaleza-CE. Enfermeiro do Hospital

Universitário da Grande Dourados-MS. fhvbqxda@yahoo.com.br;

${ }^{4}$ Especialista em Unidade de Terapia Intensiva pela Faculdade Integrada de Patos-PB, Enfermeira do Hospital Universitário da Grande Dourados-MS.
} 


\section{Introdução}

As cardiopatias tem sido um tema bastante discutido e sendo alvo de diversas pesquisas devido, principalmente, a sua alta prevalência e incidência na população mundial. Segundo Queiroga (2017), as cardiopatias representam importantes causas de internação e óbitos em crianças e o conhecimento de informações de âmbito epidemiológico são determinantes para tomada de decisão nos serviços de saúde.

As malformações congênitas assumem seu papel de destaque quando se trata de estudos epidemiológicos que, neste caso, têm-se uma grande importância no aprofundamento de conhecimentos e suas aplicabilidades no âmbito preventivo.

Segundo DATA SUS, os defeitos congênitos ocorrem em cerca de 2\% a 3\% dos recémnascidos e são os principais responsáveis pela mortalidade infantil nos países desenvolvidos. Também na América latina, essas patologias têm contribuído significativamente para a mortalidade de menores de cinco anos (BRASIL, 2016).

Grande parte das cardiopatias são de etiologias ainda desconhecidas no meio científico. Tais questões como pré-natal, idade da gestante e fatores genéticos parecem estar relacionados com a alta taxa de incidência. Contudo, estas são apenas afirmações baseadas em evidências.

A maioria dos defeitos cardíacos é de etiologia desconhecida, porém vários fatores estão associados à maior incidência, tais como: pré-natais, mãe com idade acima de 40 anos e genéticos. A prevalência de cardiopatias congênitas é nove crianças por 1000 nascidos (PINTO, 2004).

Hoje com o grande avanço tecnológico nas diversas áreas da saúde e com profissionais devidamente competentes e especializados, sabemos que as cardiopatias têm cura e que o seu diagnóstico precoce vem diminuindo a preexistência de sequelas secundárias. Este terá efetividade dos resultados, preferencialmente, se diagnosticado no primeiro trimestre de gestação por exame de imagens.

A cirurgia precoce diminui o risco de morte e as complicações que essas cardiopatias podem causar. O impacto da cardiopatia congênitas na saúde do indivíduo, na família e na sociedade é complexo, porque estas patologias são de natureza crônica e podem afetar muitos órgãos e sistemas (SILVA, 2012). Sendo assim o papel do enfermeiro no planejamento e execução de ações de cuidado a essas crianças e famílias torna-se muito importante (BOLLA, 2013). 
Diante desse contexto, surgiu a seguinte questão norteadora: qual a importância da assistência de enfermagem no âmbito de delinear estratégias do cuidado para evitar óbitos em crianças menores de cinco anos com anomalias congênitas do coração e circulatório?

Analisando o número de óbitos e fazendo uma avaliação através da Revisão Sistemática da Literatura (RSL) torna-se capaz de mostrar se existem muitos casos de óbitos com anomalias congênitas do coração e circulatório em crianças menores de cinco anos no estado do Pará em relação a nível regional e nacional. Com isso torna-se mais fácil a aplicabilidade de estratégias para melhorar a assistência de enfermagem na prevenção, com consequente melhora na evolução das crianças e redução desses óbitos. Portanto, o objetivo do presente estudo é analisar o número de óbitos por causas evitáveis, desde o ano de 2007 até 2017, sobre anomalias congênitas do coração e circulatório em crianças menores de cinco anos no estado do Pará.

\section{Revisão da Literatura}

As cardiopatias congênitas não têm causa definida, ocorrem pela interação de fatores genéticos e ambientais (PINTO, 2004). No entanto, está comprovado que existem algumas situações que podem contribuir para o aumento do risco dessa condição. Mães com mais de 35 anos, históricos de filhos anteriores cardiopatas, mães diabéticas, portadoras de lúpus e hipotireoidismo, mães que apresentaram toxoplasmose ou rubéola ou aquelas que fizeram uso de anticonvulsivos, antinflamatórios, ácido retinóico, lítio durante a gravidez podem aumentar as chances de alterações na formação do coração do feto. Gravidez de gêmeos, múltiplos ou fertilização in vitro também pode ter influência (MELO, 2010).

Segundo Queiroga (2017), o diagnóstico precoce pode salvar a vida da criança, principalmente em cardiopatias mais graves, quando o parto deve ser planejado e a criança precisa ser operada nos primeiros dias de vida. Algumas cardiopatias não necessitam de tratamento (Outras podem ser tratadas de forma eficaz com procedimentos com cateteres ou cirurgia cardiovascular. Em alguns casos podem ser necessárias várias cirurgias. Em outros, podem ser necessários transplantes de coração. Com tratamento apropriado, o prognóstico é geralmente bom, mesmo dos problemas mais complexos (SOUZA, 2008).

Existem várias causas para as principais cardiopatias congênitas. Entre elas, medicamentos, radiação, patologias como rubéola e vírus Cosakie B, e intenções de abortos (VALENTE; CIRINO, 2008). Fatores genéticos e fatores maternos, que incluem as doenças 
crônicas como a diabetes ou a fenilcetonúria mal controladas, também se destacam. Além disso, consumo de álcool, exposição a toxinas ambientais e infecções também podem aumentar consideravelmente a probabilidade de uma anomalia cardíaca.

A maioria das cardiopatias congênitas são diagnosticadas e tratadas ainda na infância, ou até mesmo logo após o nascimento. Com o advento do ecocardiograma fetal, muitas são diagnosticadas ainda na fase intra-útero (GUERRA, 2008). Este diagnóstico precoce possibilita um melhor preparo da mãe e do bebê para o nascimento. Permite também a programação de uma intervenção logo após o parto nas cardiopatias que podem levar a óbito precocemente. $\mathrm{O}$ diagnóstico pré-parto tem auxiliando na diminuição da mortalidade dos recém-nascidos com cardiopatias graves. No entanto, há casos em que a doença é identificada apenas na vida adulta. Pode também ser tratada nesta fase. Apesar dos avanços no tratamento, ainda existem doenças congênitas que levam ao óbito (GUILLER, 2007).

\section{Metodologia}

A pesquisa é do tipo qualitativa, descritivo-exploratório, foi realizado uma análise documental, contidos nas declarações de óbitos, levantados pelo Sistema de Informações sobre Mortalidade (SIM), levando em conta a unidade de federação do estado do Pará. Além disso, a amostra constituiu-se de uma avaliação crítica e a síntese de estudos publicados possibilitou conclusões relacionadas à percepção baseado nas evidências, sobre a magnitude do problema a nível estadual.

O estudo foi iniciado levando em consideração a problemática que envolve o tema, para conduzir o estudo. Este trabalho foi norteado pela seguinte questão: qual a importância da assistência de enfermagem no âmbito de delinear estratégias do cuidado para evitar óbitos em crianças menores de cinco anos com anomalias congênitas do coração e circulatório?

Logo após analisou-se o numero de óbitos segundo faixa etária infantil em menores de cinco anos / causas determinadas: anomalia congênita do coração e circulatório no estado do Pará fazendo uma comparação em relação à região norte a o Brasil. Todos os dados obtidos são segundo o Sistema de Informações sobre Mortalidade (SIM).

Por conseguinte, houve a busca na literatura para identificar a importância da assistência de enfermagem no cuidado de crianças menores de cinco com anomalias congênitas do coração e circulatório. A pesquisa foi realizada na Biblioteca Virtual de Saúde a partir dos Descritores em Ciências da Saúde (DeCS) da biblioteca virtual em saúde, utilizou na pesquisa o termo 
"Enfermagem Pediátrica" mais especificamente na Base de Dados da Literatura LatinoAmericana e do caribe em Ciências da Saúde (LILACS), Scientific Eletronic Library Online (SCIELO), Base de Dados de Enfermagem (BDENF) e Medical Literature Analysis and Retrieval System Online (MEDLINE). A escolha das bases ocorreu devido à amplitude no que tange a abrangência dos periódicos.

Foram estabelecidos como critérios de inclusão utilizados para a seleção da amostra: estudos publicados no período de 2007 a 2017, texto completo na língua portuguesa, cujo país de publicação fosse o Brasil e o tema em questão envolvesse cardiopatia congênita em menores de cinco anos. Como critérios de exclusão: artigos que não disponibilizem acesso ao texto integral.

A coleta de dados ocorreu através da análise dos resultados obtido pelo SIM e da leitura ativa dos artigos. Além do mais, realizou-se a avaliação crítica dos artigos selecionados e verificado se respondiam plenamente a pergunta-guia. Avaliaram-se todas as produções encontradas e foi analisado o papel do enfermeiro no cuidado das crianças com cardiopatia congênita. A observação crítica consistiu na fase onde todos os estudos selecionados foram avaliados com rigor metodológico.

\section{Resultados e Discursão}

Dados epidemiológicos retratam a realidade das crianças com diagnóstico de anomalia congênita do coração e circulatório, no Brasil evidenciam que nos últimos dez anos ocorreram 691 óbitos por causas evitáveis em menores de cinco anos (Tabela 1). Na análise, 89 casos de óbitos foram na região norte (Tabela 2) e o estado do Pará corresponde a 40 (5,7\%) do total de casos (tabela 3). Os dados disponíveis são oriundos do sistema de informação (SIM) obtidos através da SESPA e os resultados da pesquisa mostram que embora tenha tido uma redução de óbitos de 2007 a 2015 no Pará, houve um acréscimo do surgimento da doença, com quatro vezes mais de novos casos entre os anos de 2015 e 2016. Além disso, o estado do Pará ocupa, no ranking, o primeiro lugar $(44,94 \%)$ em relação à região norte, embora o índice seja considerado baixo se comparado aos outros estados e regiões.

Nas crianças menores de cinco anos, as mortes devido a malformações estão entre as principais causas de óbito, atrás apenas das afecções perinatais. Dentre as malformações, aquelas do aparelho circulatório são as principais componentes (GUILLER, 2007), pois essa causa muitas vezes é incompatível com a vida e altamente dependente de um adequado suporte 
médico hospitalar para a sobrevivência, levando a uma mortalidade precoce, com progressiva redução nas faixas etárias seguintes. Medidas como a realização do pré-natal e das ecocardiografias obstétricas poderiam reduzir esses óbitos, possibilitando o diagnóstico precoce e a referência dos pacientes para centros especializados de tratamento, mesmo antes do nascimento. Porém um grande problema é a rede de assistência dos sistemas de saúde pública e suplementar, não só do Estado do Pará como de todo o país.

Em decorrência dos avanços tecnológicos para melhoria nas condições de nascimento e de assistência a saúde infantil, está ocorrendo um aumento na sobrevida de crianças que nascem com algum tipo de anomalia congênita.

\begin{tabular}{|c|c|c|}
\hline \multirow[t]{2}{*}{ Ano } & \multirow[t]{2}{*}{ Numero de casos } & \\
\hline & & $\%$ \\
\hline 2007 & 77 & 11,14 \\
\hline 2008 & 90 & 13,02 \\
\hline 2009 & 89 & 12,88 \\
\hline 2010 & 52 & 7,52 \\
\hline 2011 & 81 & 11,72 \\
\hline 2012 & 69 & 9,98 \\
\hline 2013 & 45 & 6,51 \\
\hline 2014 & 65 & 9,40 \\
\hline 2015 & 59 & 8,53 \\
\hline 2016 & 64 & 9,30 \\
\hline 2017 & Não informado & - \\
\hline Total & 691 & 100 \\
\hline
\end{tabular}

Fonte: SIM (sistema de informação)

Tabela 2- óbitos por causas evitáveis em menores de 5 anos / causas determinadas: anomalia congênita do coração e circulatório Na região Norte, segundo o Sistema de Informações sobre Mortalidade (SIM).

\begin{tabular}{|c|c|c|c|c|c|c|c|c|c|c|c|c|}
\hline $\begin{array}{l}\text { Região/ } \\
\text { Unidade de } \\
\text { federação }\end{array}$ & 2007 & 2008 & 2009 & 2010 & 2011 & 2012 & 2013 & 2014 & 2015 & 2016 & 2017 & Total \\
\hline Rondônia & 1 & 1 & 1 & - & 1 & - & 1 & - & - & 1 & $*$ & 6 \\
\hline Acre & 2 & 1 & 1 & 1 & - & 1 & - & - & - & - & $*$ & 6 \\
\hline Amazonas & - & - & 3 & 1 & - & 1 & 2 & 1 & 2 & 2 & $*$ & 12 \\
\hline Roraima & - & 1 & 1 & 1 & - & 1 & - & 1 & 4 & - & $*$ & 9 \\
\hline Pará & 3 & 1 & 9 & 2 & 4 & 6 & 5 & 5 & 1 & 4 & $*$ & 40 \\
\hline Amapá & - & 1 & 3 & 2 & 2 & - & - & - & - & - & $*$ & 8 \\
\hline Tocantins & 1 & - & 1 & - & 3 & 2 & - & 1 & - & - & $*$ & 8 \\
\hline $\begin{array}{l}\text { Região } \\
\text { Norte/ } \\
\text { Total }\end{array}$ & 7 & 5 & 19 & 7 & 10 & 11 & 8 & 8 & 7 & 7 & $*$ & 89 \\
\hline
\end{tabular}

Fonte: SIM (sistema de informação) 
Tabela 3 - óbitos por causas evitáveis em menores de 5 anos / causas determinadas: anomalia congênita do coração e circulatório no estado do Pará, segundo o Sistema de Informações sobre Mortalidade (SIM).

\begin{tabular}{ccc}
\hline Ano & Numero de casos & \% \\
\hline $\mathbf{2 0 0 7}$ & 3 & 7,5 \\
$\mathbf{2 0 0 8}$ & 1 & 2,5 \\
$\mathbf{2 0 0 9}$ & 9 & 22,5 \\
$\mathbf{2 0 1 0}$ & 2 & 5 \\
$\mathbf{2 0 1 1}$ & 4 & 10 \\
$\mathbf{2 0 1 2}$ & 6 & 15 \\
$\mathbf{2 0 1 3}$ & 5 & 12,5 \\
$\mathbf{2 0 1 4}$ & 5 & 12,5 \\
$\mathbf{2 0 1 5}$ & 1 & 2,5 \\
$\mathbf{2 0 1 6}$ & 4 & 10 \\
Total & 40 & 100 \\
\hline
\end{tabular}

Fonte: SIM (sistema de informação)

$\mathrm{Na}$ análise da literatura baseada em evidências observou que a importância da assistência de enfermagem no cuidado de crianças menores de cinco com anomalias congênitas do coração e circulatório possui uma tarefa desgastante do ponto de vista emocional, mas capaz de gerar grande satisfação pessoal (QUEIROGA, 2017), uma vez que quando há trabalho em equipe, aliado ao conhecimento teórico, as dificuldades podem ser minimizadas e superadas com maior facilidade. No caso de cirurgias. Quando o enfermeiro estimula a mãe a falar sobre a situação, torna-se mais fácil ajudá-la a encontrar respostas às suas dúvidas, o significado para a mãe de ter um filho com problema no coração.

Assim sendo, entende - se que os planos de assistência para mãe e filho requerem um repensar, no qual condutas de cuidado estejam voltadas às necessidades vivenciadas pela mãe, valorizando a maneira como ela percebe a situação (SILVA, 2012), pois, na procura de aperfeiçoamento, a equipe de enfermagem busca direcionar e integrar saber comfazer, contribuindo para melhoria da qualidade da assistência.

Faz-se necessário um programa de educação continuada interno (BOLLA, 2013), pois para que todos profissionais envolvidos no atendimento dessas crianças possam atuar com conhecimento dos processos fisiopatológicos envolvidos nas cardiopatias congênitas e suas correções. Sobretudo, devem-se criar propostas de intervenção centradas em prevenção e tratamento, assim como de protocolos assistenciais para o cuidado. Com base nas reflexões, ressalta-se que a qualidade da assistência ao paciente resulta em uma crescente participação da equipe de enfermagem. 


\section{Conclusões}

Existem poucos estudos em nosso Estado sobre taxas de óbitos neonatais por cardiopatias congênitas. Trabalhos com esta temática são de grande relevância, pois permitem a produção de novos conhecimentos para a melhoria da assistência e propor intervenções centradas no diagnóstico precoce e tratamento em tempo hábil para a redução dos índices de mortalidade. Para a enfermagem, informações acerca da patologia e de seus impactos nos indicadores de mortalidade são fundamentais, permitindo ao profissional a construção de uma assistência focada nas necessidades humanas básicas alteradas pela cardiopatia congênita, de maneira humanizada e holística e contribuindo para a melhora do prognóstico da doença.

Atualmente existem ações governamentais relacionadas à prevenção e a monitorização dos defeitos congênitos. Além da triagem neonatal e tratamento pode-se auxiliar sobremaneira a programação do atendimento e prevenção da cardiopatiacongênitacomo a fortificação da farinha do ácido fólico, imunizações e declaração de recém-nascidovivo (DN)- documento oficial emitido pelas maternidades. Acredita-se ser de grande importância a realização de pesquisas de enfermagem com famílias que vivenciam tal situação, a fim de melhor compreender sua experiência no processo de cuidar da criança em seu cotidiano. Porém, é preciso investirmais em pesquisas relacionadas a intervenções, a fim de instrumentalizar o enfermeiro para o cuidado e ampliar o corpo de conhecimento teórico da área de enfermagem da família fundamentado em evidências científicas. Promover melhorias no sistema público de atendimento à criança cardiopata não é um problema isolado do Governo, o enfermeiro como especialistas no assunto, deve estar lado a lado, juntamente com a sociedade em geral, nesse esforço conjunto de melhorias.

\section{Referências}

BRASIL. MINISTÉRIO DA SAÚDE- DATASUS - Tecnologia da informação a serviço do SUS. 2016.

BOLLA, B.A.; FULCONI, S.N.; BALTOR, M.R.R.; DUPAS, G. Cuidado da criança com anomalia congênita: a experiência da família. Esc. Anna Nery vol.17 no. 2 Rio de Janeiro Apr./June 2013. Disponível em:<http://www.scielo.br/pdf/ean/v17n2/v17n2a12.pdf >.

GUERRA, F.A.R.; LLERENA JR, J.C.; GAMA, S.G.N.; CUNHA, C.B.; THEME FILHA, M.M. Confiabilidade das informações das declarações de nascido vivo com registro de defeitos 
congênitos no Município do Rio de Janeiro, Brasil, 2004. Cad. Saúde Pública, Rio de Janeiro , v. 24, n. 2, p. 438-446, Feb. 2008 . Available from <http://www.scielo.br/scielo. php?script=sci_arttext\&pid=S0102-311X2008000200023\&lng=en\&nrm=iso>.

GUILLER, C.A.; DUPAS, G.; PETTENGILL, M.A.M. Criança com anomalia congênita: estudo bibliográfico de publicações na área de enfermagem pediátrica. Acta paul. enferm., São Paulo, v. 20, n. 1, p. 18-23, Mar. 2007 . Available from <http://www.scielo. br/scielo.php?script=sci_arttext\&pid=S0103-21002007000100004\&lng=en\&nrm=iso $>$.

MELO, W.A.; ZURITA, R.C.M.; UCHIMURA, T.T.; MARCON, S.S. Anomalias congênitas: fatores associados à idade materna em município sul brasileiro, 2000 a 2007 . Rev. Eletr. Enf. [Internet]. 2010; 12(1): 73-82. Disponível em: < https://www.fen.ufg.br/revista /v12/n1/pdf/v12n1a09.pdf > Acesso em 02/08/2018.

PINTO JÚNIOR, V.C.; DAHER, C.V.; SALLUM, F.S.; JATENE, M.B.; CROTI, U.A. Situação das cirurgias cardíacas congênitas no Brasil. Rev Bras Cir Cardiovasc. 2004;19(2):III-VI

QUEIROGA, A.V.; ARAÚJO, H.V.S.; BELO, R.M.O.; FIGUEIRÊDO, T.R.; BEZERRA, S.M.M.S. Estratégias educativas para redução da ansiedade dos cuidadores de crianças com cardiopatia congênita. Rev Fun Care Online. 2017 out/dez; 9(4): 1061-1067. Disponível em: < http://dx.doi.org/10.9789/2175-5361.2017.v9i4.1061-1067>. Acesso em : 13/08/2018.

SILVA, G.V. Protocolo de cuidados de enfermagem para crianças com cardiopatias congênitas: uma proposta baseada em NANDA. Dissertação (Mestrado Profissional em Enfermagem Assistencial) - Universidade Federal Fluminense, 2012. Disponível em: <https://app.uff.br/riuff/handle/1/1446> . Aceso em : 21/09/2018.

SOUZA, P.; SCATOLIN, B. E. A relação da equipe de enfermagem com a criança e a família em pós-operatório imediato de cardiopatias congênitas; Arq. Ciências e Saúde. SP. 2008

VALENTE, A.S.; CIRINO, C.M.F. Cardiopatia congênita no adulto. In: Croti UA, Mattos SS, Pinto Jr VC, Aiello VD, eds. Cardiopatia e cirurgia cardiovascular pediátrica. São Paulo:Roca; 2008.

\section{Como citar este artigo (Formato ABNT):}

SILVA; Eliane Moura da; OLINDA, Antônia Gomes de; BARROS, Francisco Hilângelo Vieira; ROSENO, Maria Alcione Silva Gomes. Óbitos por Anomalias Congênitas do Coração e Circulatório no Estado do Pará nos anos de 2007 a 2017: Revisão Sistemática da Literatura. Id on Line Rev.Mult. Psic., 2018, vol.12, n.42, Supl. 1, p. 823-831. ISSN: 1981-1179.

Recebido: $14 / 11 / 2018$

Aceito: 17/11/2018 